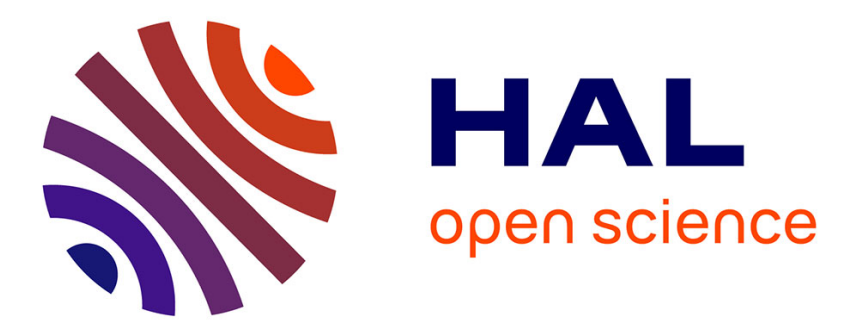

\title{
COMPORTEMENT AUX CHOCS PAR EXPLOSIONS SOUS-MARINES DE PLAQUES EN COMPOSITES VERDE-RÉSINE
}

J. Picard, P. Seris

\section{- To cite this version:}

J. Picard, P. Seris. COMPORTEMENT AUX CHOCS PAR EXPLOSIONS SOUS-MARINES DE PLAQUES EN COMPOSITES VERDE-RÉSINE. Journal de Physique IV Proceedings, 1991, 01 (C3), pp.C3-361-C3-367. 10.1051/jp4:1991352 . jpa-00250496

\section{HAL Id: jpa-00250496 https://hal.science/jpa-00250496}

Submitted on 1 Jan 1991

HAL is a multi-disciplinary open access archive for the deposit and dissemination of scientific research documents, whether they are published or not. The documents may come from teaching and research institutions in France or abroad, or from public or private research centers.
L'archive ouverte pluridisciplinaire HAL, est destinée au dépôt et à la diffusion de documents scientifiques de niveau recherche, publiés ou non, émanant des établissements d'enseignement et de recherche français ou étrangers, des laboratoires publics ou privés. 


\title{
COMPORTEMENT AUX CHOCS PAR EXPLOSIONS SOUS-MARINES DE PLAQUES EN COMPOSITES VERRE-RESINE
}

\author{
J.M. PICARD et P. SERIS \\ STCAN, 8, Bd Victor, F-75732 Paris cedex 15, France
}

\begin{abstract}
In this paper, the effects of underwater explosions on GRP plates are studied. Trials are described. A simple energetical model built up for naval architects is showed and compared to measurments and to tinite elements high dynamics calculations. A tracture criteria is given.
\end{abstract}

\section{DNTRODUCTION}

La but de cette énde est de mener une première investigation visant à mieux comprendre les elfets d'une explosion sous-marine sur une plaque en composite. Il ne s'agit pas d'un test de validation, ni d'une comparaison de performances. On désire connaître le mode de dégradation de la structure et mieux cemer les mécanismes de dégradacion.

Les essais sur éprouvettes permettent de déterminer les caractéristiques mécaniques de ces matériaux. Dans cette étude, l'approche est différente. Nous nous intéressons à la réponse et à la modélisation d'une suructure simple soumise a un véritable chargement par explosions.

Le matériau choisi est celui employé pour la consunction de la coque du Bâtiment Anui-Mines Océaniques. Il s'agit d'un composite verre-résine tait d'un empilement de ussus de verre $\mathrm{E}$ sergé de $2,0^{\circ}, 90^{\circ}, 830 \mathrm{~g} / \mathrm{m} 2$. Chaque pli est appiiqué au rouleau et fait environ $1 \mathrm{~mm}$ d'épaisseur. Les ussus ont mème orientation, la résine est polyester isopintalique et polymérise à l'ambiante.

Après avoir présenté les essais, nous nous efforcerons d'interpréter leurs résultais au travers de diverses modéiisations.

\section{i - LES ESSAIS DEXPLOSIONS SOUS-MARINES}

\section{I.1 - Description des essais}

Las essais ont lieu en piscine calibre a 4 g de TVT. La cioble est constitue diune plaque circulaire en composite verre-résine de diamètre $320 \mathrm{~mm}$, montée au sommet d'un support métallique cylindrique (tumbour). Line couronne métallique permet un encastrement de la plaque sans perçage. Ce montage élunche maintient la face intérieure an jir. Lies tirs d'une charge de $+\mathrm{g}$ ont lieu en eau sur l'axe vertical de la plaque.

L'instrumenution consiste en jauges de déformation es en accéléromèures montés sur la lìce en air de ha olauue. L'n témoin de pâte à mocieler donne la tlèche maximale au cenure.
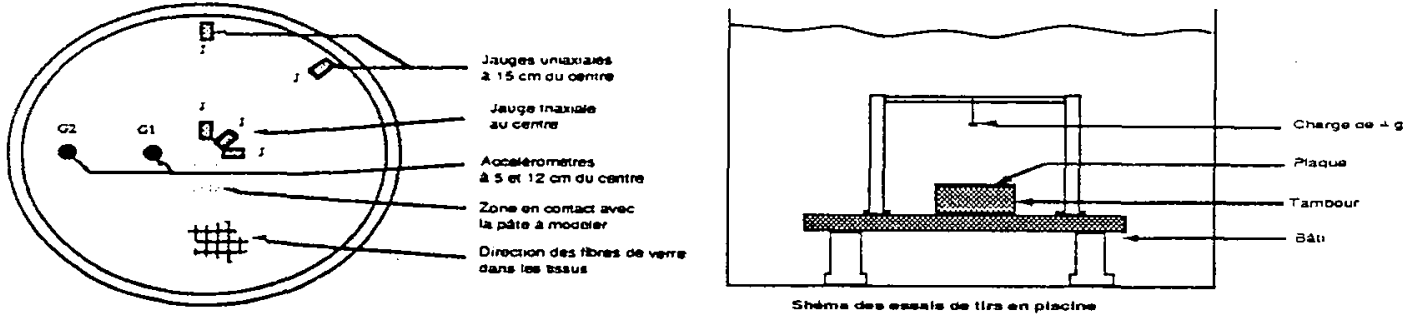


\section{2 - Observations qualitatives}

Une vingtaine de plaques de deux épaisseurs à quatre et huit plis a été essayéc. Chaque plaque naa subi qu'un seul tir. Pour.des distances de tir décroissantes, les dommages suivants ont été obscrvés.

STADE I Fissuration superficielle de la résine sur la face en extension de la plaque. La résine se fendille en surface selon des directions orthogonales aux fibres de verre

STADE II Délaminage à la périphérie de la parte plane de l'ćprouvetre, visible sur la partic extérieure au niveau du rebord.

ST ADE III Couronne de délaminage au centre de la plaque.

ST ADE IV Rupture partielle ou totale de la plaque par cisaillement à la périphérie.

\begin{tabular}{|l|l|l|}
\hline & Q4 & P8-Q8 \\
\hline Stade I & $600 \mathrm{~mm}$ & $600 \mathrm{~mm}$ \\
\hline Stade II & $400 \mathrm{~mm}$ & $400 \mathrm{~mm}$ \\
\hline Stade III & $?$ & $200 \mathrm{~mm}$ \\
\hline Stade IV & $250 \mathrm{~mm}$ & $100 \mathrm{~mm}$ \\
\hline
\end{tabular}

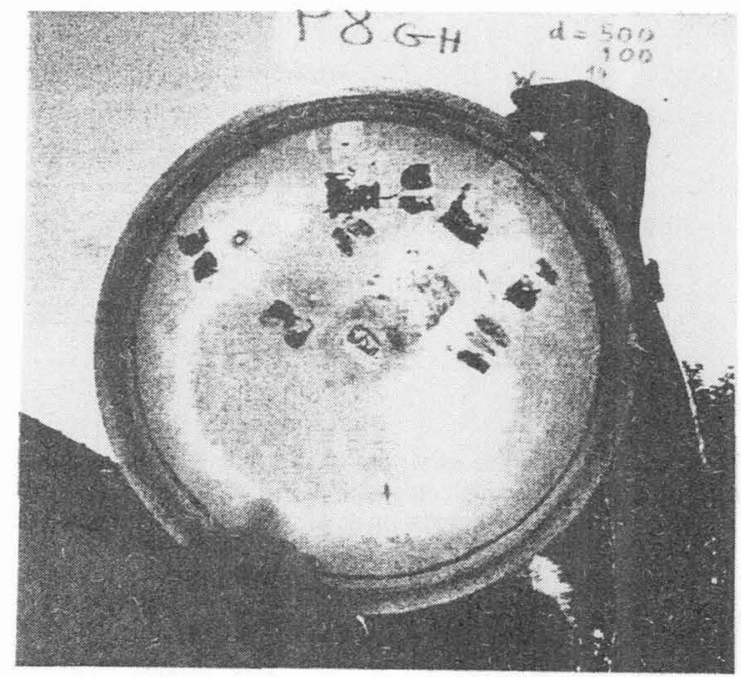

Le tableau ci-dessus donne les distances de tir correspondant à l'apparition des différents stades. Malgré l'endommagement, aucune déformation résiduelle autre que la rupture n'a été relevée.

\section{I.j - Résultats quantitatifs}

Lélude quantitative a été faite sur les plaques les plus épaisses, à huit plis. Elles proviennent de deux lots de tabrication différents, notés $P$ et $Q$.

Les signaux bruts foumis par les accéléromètres sont difficilement exploitables. En effet les accélérations initiales de plusieurs dizaines de milliers de g ne rendent pas compce du mouvement d'ensemble de la plaque. En revanche. l'intégration de ces signaux montre une mise en vitesse quasi-instantanée et a priori uniforme de la plaque : la vitesse maximale de 10 à $12 \mathrm{~m} / \mathrm{s}$ est atteinte en 50 à $90 \mu \mathrm{s}$.

Trois jauges sont collées au centre, deux à la péripherie. Il niy a pas de différences dans l'allure du signal suivant la direction des jauges. Les trois jauges au centre répondent de la mème façon et il cn est de mème pour les jauges j la périphérie. Les différences constatées au niveau de l'amplitude de la réponse sont de l'ordre de $10 \%$, ce qui correspond aux incertitudes sur les mesures. Nous niavons pas d'effets notables d'anisotropic.

Les oscillations lentes (environ $110 \mathrm{~Hz}$ ) proviennent de la vibration en eau des plaques. Les oscillations rapides $(285 \mathrm{~Hz})$ sont dues soit à une harmonique, soit a une vibration sans effels dus a l'eau lorsqu'il y a cavilation au dessus de la plaque.

Les signaux de jauges lont apparâture deux pics principaux, le deuxième étant légèrement supérieur. Le premier correspond à l'onde de choc primaire, le deuxième à l'onde de pression seccondaire émise par la première contraction de la bulle des gaz créée par l'explosion. 

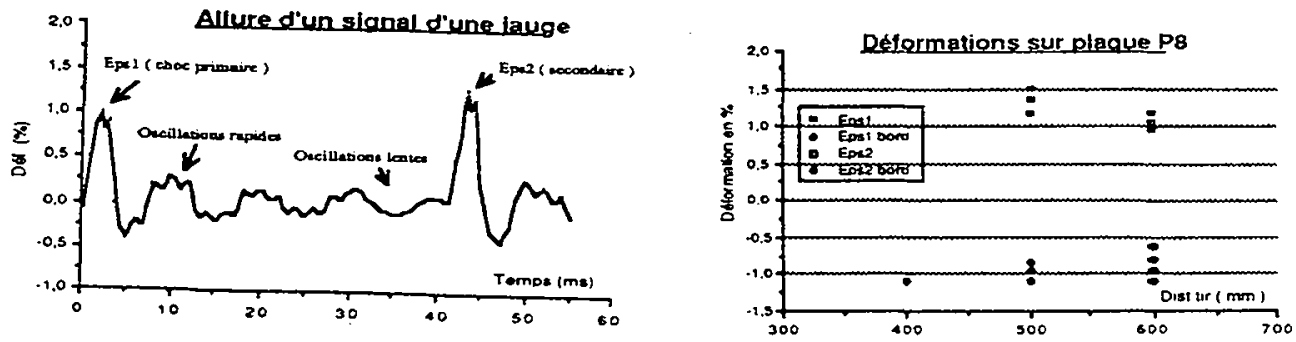

Les flèches maximales relevées sont rassemblées sur la figure ci-dessous :

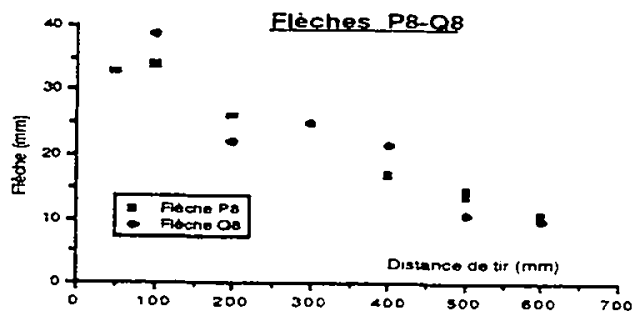

On constate que les flèches maximales aneignent quarre fois l'épaisseur des plaques et le dixième de leur diamètre. La modélisation devra prendre en compte les effets de membrane.

\section{LES MODELISATIONS.} métalliques.

La démarche initiale a consisté à utiliser une approche énergétique simpie mise au point sur des essais aver plaques

\section{Il.1 - Choix des paramères.}

Comme le suggèrent les résultats des essais et le modèle de la plaque métallique, nous considérons que l'onde de pression communique essentiellement une impulsion à la plaque. Celle-ci acquiert une vitesse uniforme alors que les déplacements sont encore minimes. Le mode de fixation de la plaque ou le type de matériau utilisé imponte peu dans cette approche, seul compte la masse volumique de l'élément de plaque considéré.

Le paramètre qui guide ce raisonnement est la constante de temps de l'onde. Celle ci est très petite, de l'ordre de $20 \mu \mathrm{s}$. Pendant ce temps, une onde acoustique se déplace de $3 \mathrm{~cm}$ dans l'eau et à peine plus dans le matériau composite. Il est donc légitime de penser que le mouvement initial de la plaque est peu influencé par son mode de fixation au bord.

Les paramètres retenus pour décrire le chargement sont donc les impulsions de l'onde de choc primaire et de la première onde de pression secondaire. Elles sont données par des abaques connues.

Tant que le matériau n'est pas endommagé, le paramèure qui intéresse l'architecte naval est la flèche maximale atteinte. On peut relier celle-ci à l'impulsion initiale au travers de l'énergie de déformation élastique dans la déformée réelle, que l'on approche par la déformée sous pression statique uniforme. Les contraintes calculées par ce procédé sont comparées aux critères d'endommagement. 
DEPLACEMENT cm

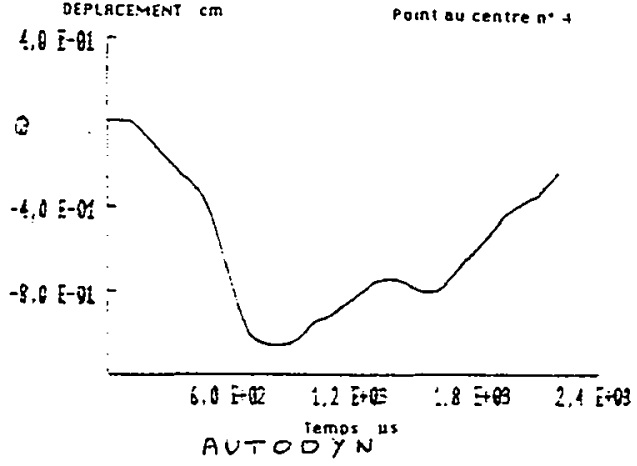

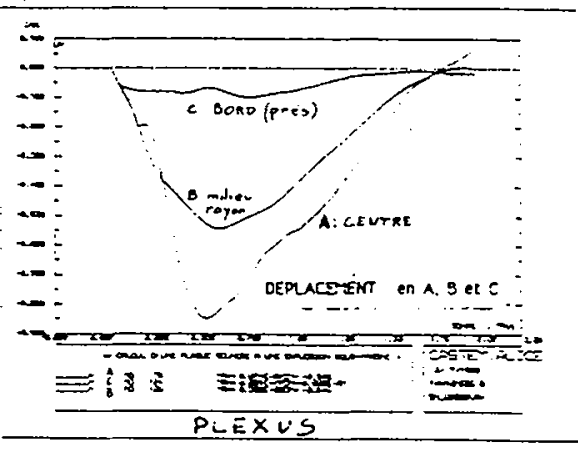

III - COMPARAISON ESSAIS-MODELES.

III.1 - Relation flèche-énergie.

La modélisation retenue foumit une relation entre la flèche maximale au centre et l'énergie fournie à la plaque de la forme:

$$
\text { Energie }=\mathrm{A}(\text { flèche })^{2}+B(\text { flèche })^{4}
$$

A et $\mathrm{B}$ dépendent de la géométrie de la plaque, des conditions aux limites el du matćriau. A est relié au terme de flexion, $B$ à celui de membrane.

En supposant un encastrement parfait et en utilisant les caractérisuques du matériau données par le fabricant d'après des essais sur éprouvettes, on obuient la courbe A qui correspond à notre premier modèle.

Au vu des résultats des essais en caisson hyperbare, on constate que les coefficients $A$ et $B$ calculés précédemment sont incorrects. On peut mettre en cause un défaut dencasurement ou une imprécision sur les caractéristiques du matériau. La courbe B est ajustée sur les essais statiques.

Les essais d'explosions sous-marines fournissent également une relation flèche-énergie représentée par la courbe $C$. Cette dernière courbe correspond à des mesures dynamiques alors que les autes s'appuient sur des données en statique.

II subsiste un écart enure les courbes $B$ et $C$. Il semblerait que lion mette en évidence les effets de l'endommagement du matériau au cours des essais d'explosions.

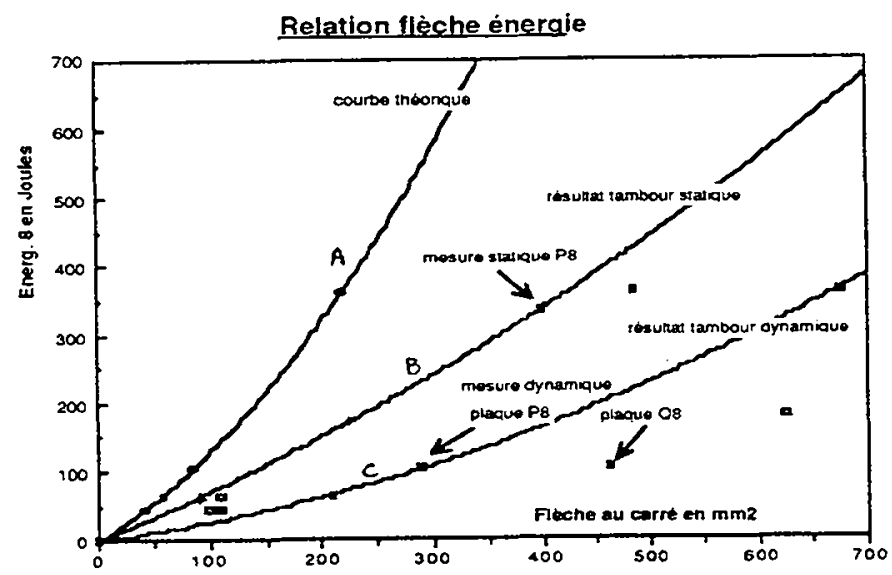

III.2 - La ruprure.

La npture a l'aliure d'un cisaillement. Nous allons rechercher la contrainte de cisailiement équivalente, en supposant que cette demière est constante à travers l'épaisseur h de la plaque à sa périphérie.

Soit $P_{\text {eq }}$ la pression donnant la même énergie de déformation à la plaque en statique. La force transversale appliquée à la plaque est $P_{e q} \cdot \pi a^{2}$, où a est la surface de la plaque. Le cisaillement $\tau$ à la périphérie permettant d'équilibrer cette force vérifie :

$$
\tau . h .2 \pi a=P_{\text {eq }} \pi a^{2}
$$




\section{II.2 - Lo modèje analytique.}

Un modèie simpie represente linteraction de londe de choc avec la piaụue: la rćflexion cuune onòe piane de mèmes paramètres sur une plaque inñnie de mème masse surfacique qui sípare leau de lair.

Ce probième a une soiution anał tique. Soient:

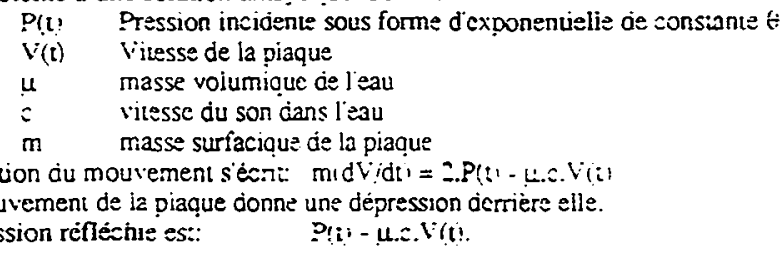

L'onde de choc peus se représenter par une exponenuclie de consiante de temps e. La vilesse passe par un

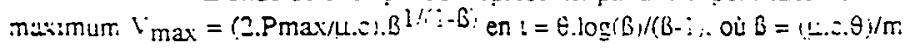

Dans les applications numérig̣ues. fo est de loröre je "unité. la vitesse maximale esi auteinte en un temps comparable à celui de $\theta$. Lorsque la piaque attein: la vilesse lmax. ia pression derrière la plaque s annuie e: dtenć à davenir

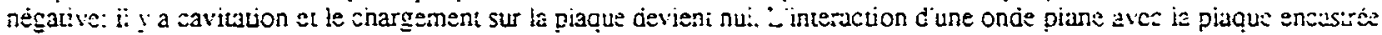
sst jonc modélisée par une mise en vilesse insiantané é unijorme de la piague entiers.

Si le tur esi procine. ̈onde de pression n esi pius piane mais spnerrique. : impuision communıcuée a es: pius identique en tout poinc. Les caizurs lies à ces tirs conduisent à des corrections sur l'énerşie cinétique communiquée i ia piaque.

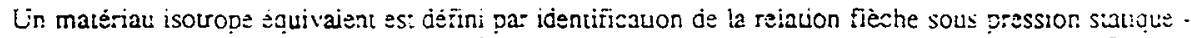

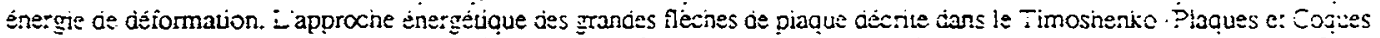

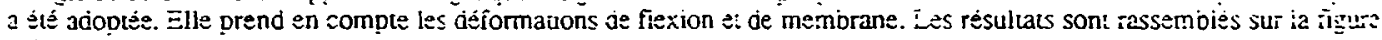
suivante.

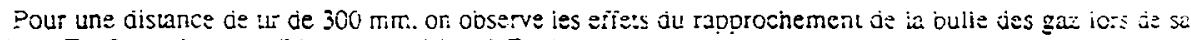

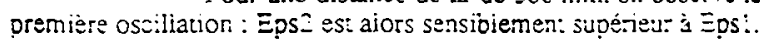

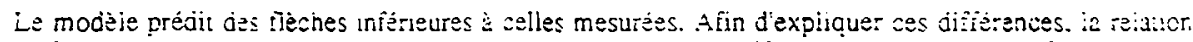

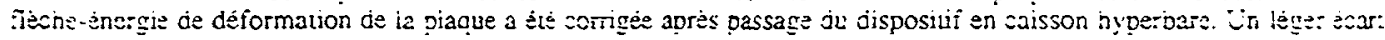
sucsisio enure modèle ei essais.

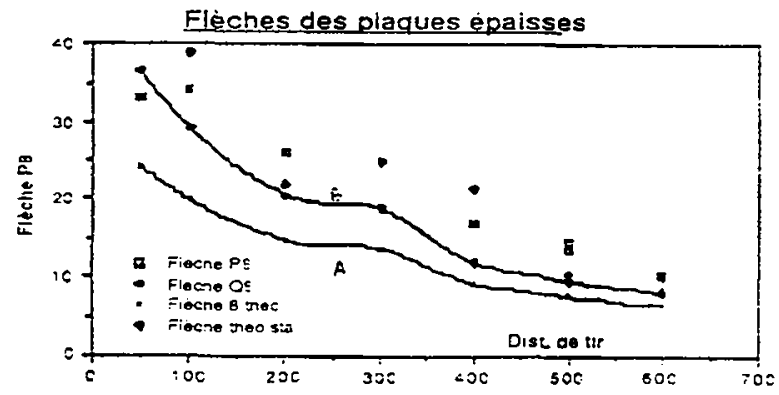

\section{İ:.: - Les modèies numériques.}

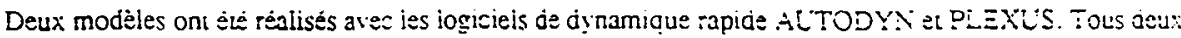
prennent en compte les interactions fluide-soucture et les effets de la zavitation par minoration de la pression par zér. ¿es zaleuis ont Été jait en axisymétriọue sư une coionne deau sumontant une piaque encastrée. Une onde de pression de méme

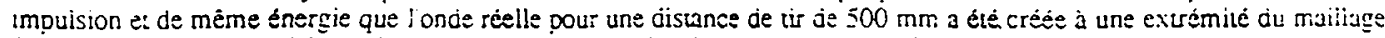
ituidz. Les vitesses impuisionnelles sont bien retrouvées. les dépiacement restent légèremeni inférieurs. 
Sur la courbe ci-dessous, nous avons reporté ces valeurs du cisaillement en fonction de la distance de tir. Si l'on suppose que le cisaillement a un profil parabolique dans l'épaisseur, sa valeur maximale est obtenue en multipliant ces courbes par 1,5 .

La valeur limite du cisaillement mesurée sur des éprouvettes est:

(dans une hypothèse parabolique)

4 couches $\quad 45$ à $49 \mathrm{MPa}$

9 couches $\quad 42$ à $44 \mathrm{MPa}$

Une autre méthode d'essai (méthode IOSIPESCU) donne les résultats suivants :

4 couches $\quad 58 \mathrm{MPa}$

9 couches $\quad 72$ a $78 \mathrm{MPa}$

La rupture par explosion est indiquée sur la figure. Le cisaillement auteint est du même ordre de grandeur que les données sur éprouvettes. Ainsi l'hypothèse d'une rupture par cisaillement semble la plus probable. Cependant, il ne faut pas oublier que les tensions de membrane et de flexion sont également très fore lors de la rupture. La rupture est due à la combinaison de ces trois effets.

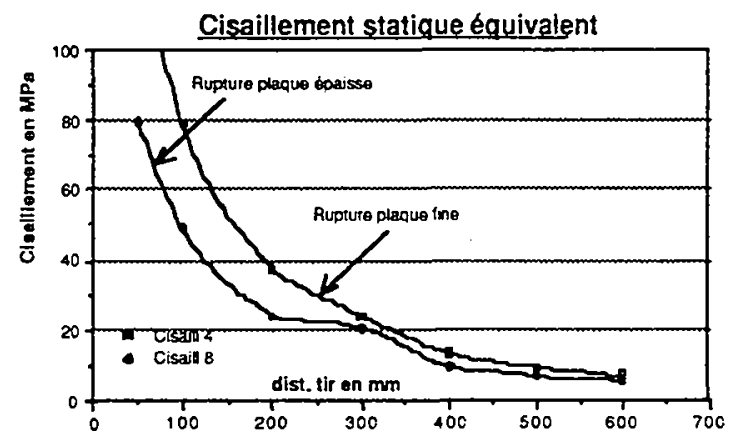

\section{CONCLUSION}

Les essais d'explosions sous-marines ont pour but de mieux connaitre les mécanismes de dégradation spécifiques d'une structure navale, afin d'améliorer la conception et le dimensionnement de navires avec une coque en composite. Ce type d'expériences a l'avantage sur les essais sur éprouvettes de soumettre le matériau au chargement réel, tout en restant relativement facile de mise en cuvre et pcu onéreux. En revanche, son interprétation détaillée s'avère plus complexe. Il permet cependant d'élaborer des modèles globaux, tels que celui présenté ici, accessibles aux bureaux d'études. 
BLAZYNSKI T. Z. : Materials at high strain rates Elsevier Applied Science

COLE Robert H. : Underwater explosions Princeton University Press 1948

HENRYCH Josef : The dynamics of explosion ELsevier 1982

KENNARD E. H. : Migration of underwater gas globes due to gravity and neighboring surfaces. (1943)

S. LAROZE, J.J. BARRAU : Calculs des structures en matériaux composites Mécaniques des strcures, Tome 4 Eyrolles Masson. 1987

M. CALEDEC, X. GUILPIN : Projet de fin d'études ENSTA (1986 STCAN) Comportement de bordés de fond de navire soumis à une explosion sous-marine

Note Technique $N^{\circ} 115 / 85$ Gerpy Etude à échelle réduite des effets d'une explosion sous-marine.

Note Technique $N^{\circ} 70 / 86$ Gerpy Considération énergétique et étude du collage de la bulle.

Note Technique $N^{\circ} 1570 / 88$ Gerpy Eléments pour la détermination de la mise en vitesse de bordées de navires lors des explosions sous marines.

Note Technique $N^{\circ} 108 / 89$ Gerpy

Analyse numérique de la réponse de plaques circulaires à comportement élasto-plastique sous l'effet d'une onde de choc sous marine.

CAPRINO G., CRIVELLI VISCONTI I., DI IIIO A. : "Composites materials responses under low velocity impact" Composites Siructures 2 (1984) 261-271

LAL K. M. : "Low velocity transverse impact behavior of 8 ply, Graphite-Epoxy laminates" Journal of Reinforced Plastics and Composites 2 (oct 83) 216-225

LAL K. M. : "Residual strength assesment of low velocity impact damage of Graphite-Epoxy laminates" Journal of Reinforced Plastics and Composites 2 (oct 83) 216-225

BEAUMONT N., PENAZZI L., CAZENEUVE C. : "Etude de l'impact d'une bille sur une plaque composite à tibres longues et à matrice organique" Compte rendu de la $5^{\circ}$ joumée nationale DYMAT 